Questions vives

\section{Questions Vives}

Recherches en éducation

$\mathbf{N}^{\circ} 34 \mid 2020$

Expériences vécues et surgissement d'événements : une écriture du sensible en recherche biographique

\title{
Cadre éthique et innovation méthodologique : chercher avec des personnes en situation de maladie auto-immune rare
}

Methodological innovation in research with people with rare autoimmune disease

\section{Anne Dizerbo}

\section{(2) OpenEdition}

Journals

\section{Édition électronique}

URL : https://journals.openedition.org/questionsvives/5029

DOI : 10.4000/questionsvives.5029

ISSN : 1775-433X

Éditeur

Université Aix-Marseille (AMU)

\section{Édition imprimée}

Date de publication : 18 décembre 2020

ISBN : 978-2-912643-58-2

ISSN : 1635-4079

\section{Référence électronique}

Anne Dizerbo, «Cadre éthique et innovation méthodologique : chercher avec des personnes en situation de maladie auto-immune rare », Questions Vives [En ligne], №34 | 2020, mis en ligne le 18 décembre 2020, consulté le 11 octobre 2021. URL : http://journals.openedition.org/questionsvives/ 5029 ; DOI : https://doi.org/10.4000/questionsvives.5029

Ce document a été généré automatiquement le 11 octobre 2021.

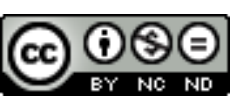

Questions Vives est mis à disposition selon les termes de la licence Creative Commons Attribution -

Pas d'Utilisation Commerciale - Pas de Modification 4.0 International. 


\title{
Cadre éthique et innovation méthodologique : chercher avec des personnes en situation de maladie auto-immune rare
}

\author{
Methodological innovation in research with people with rare autoimmune \\ disease
}

Anne Dizerbo

\section{Introduction}

1 Les pathologies chroniques, devenant plus nombreuses et visibles, rendent nécessaire un nouveau type d'approche de la prise en charge de la maladie. Celui-ci remet en question l'organisation verticale et cloisonnée des services de santé et la façon de travailler des professionnels. La maladie chronique introduit en effet des changements importants dans le quotidien des personnes qui en sont atteintes (dans leurs routines, leurs relations interpersonnelles, leur vie professionnelle et leur rapport aux institutions médicales). Ces changements impliquent de prendre en compte les personnes atteintes de maladie chronique dans la globalité de leur parcours et «il s'ensuit que leurs représentations, leurs attentes, font désormais légitimement partie de ce que les professionnels doivent prendre en compte pour construire leurs interventions " (Mazereau, 2016, p.63). Cela implique une coopération accrue des professionnels de la santé avec les malades et leurs familles ainsi qu'un partage entre des savoirs issus de l'expérience de vivre avec la maladie et des savoirs scientifiques émanant des recherches médicales (Jouet, Flora, Las Vergnas, 2010), qui s'altèrent réciproquement pour permettre la construction d'un savoir nouveau in situ (Mazereau, Ibid). 
2 Dans cette perspective, les recherches en santé s'appuyant sur la subjectivité et l'expérience des malades, d'abord développées dans les pays anglo-saxons, ont fait leur apparition en France alors que les plans nationaux «Maladies Rares » successifs de 2005-2008 et de 2011-2014 soulignaient l'importance d'analyser la qualité de vie et les handicaps rencontrés par les patients atteints d'une maladie auto-immune rare pour contribuer à l'amélioration de leur prise en charge. Il en a découlé le développement de recherches innovantes s'appuyant sur l'analyse des savoirs expérientiels. Elles s'efforcent parfois de dépasser les clivages et les hiérarchisations traditionnelles entre les différentes formes de savoirs en proposant aux malades un statut de co-chercheurs, notamment, à partir de 2010, dans des investigations concernant certaines maladies chroniques (cancer, dépression) (Flora, 2011, pp. 239-253).

3 C'est dans cette perspective que s'est inscrite la recherche qualitative s'ancrant dans le domaine des sciences de l'éducation et de la formation dont il sera question, qui concernait des personnes atteintes de deux maladies systémiques auto-immunes rares : le lupus et la sclérodermie. L'anthropologue Éric Chauvier (2012) pose la question de la violence symbolique exercée par le chercheur quand il dénie la position d'interlocuteur de l'observé dans une situation qu'il propose de nommer « désinterlocution ». Il met en évidence les paradoxes soulevés par la volonté de donner voix aux acteurs, notamment dans le cadre de l'observation du «déclassement social» (p. 213) que le "processus d'observation lui-même » peut potentiellement venir « renforcer » (p. 212).

4 La recherche sur laquelle s'appuiera notre propos, concernant des personnes atteintes de lupus et de sclérodermie, s'inscrivait dans une conscience de ces paradoxes et espérait les dépasser en créant un espace d'interlocution où co-construire les connaissances. Cette intention n'a malheureusement pas permis finalement de faire entendre la parole des sujets, venant interroger le cadre éthique de cette investigation, alors même qu'elle s'efforçait de comprendre et restituer un savoir sensible: "Le sensible est ce qui est perceptible, tangible, visible, mais aussi phénoménal. Il permet de s'orienter dans l'existence et de mettre en perspective ce qui advient ou est advenu dans notre vie. Le sensible relève ainsi de la subjectivité (Baeza, 2019, p. 161). De cette recherche, il ne sera pas possible de nommer le commanditaire (une fondation indépendante des équipes de chercheurs), les responsables des équipes ayant collaboré pour répondre à son appel à projet, ou d'en mentionner les résultats. Au terme de deux années, elle s'est en effet achevée par la transmission au commanditaire d'un rapport contenant les résultats. Cependant des difficultés, révélatrices de certains enjeux et écueils des recherches s'inscrivant dans une perspective anthropologique et visant à donner la parole à des personnes en situation de vulnérabilité, en ont interdit la diffusion scientifique.

5 Les échecs en recherche qualitative sont le plus souvent passés sous silence, dans un contexte où les laboratoires, mis en concurrence, doivent faire preuve de leurs bons résultats, et où la recherche qualitative se trouve parfois mise en demeure de légitimer sa scientificité :

Les sciences anthroposociales [...] sont remises en cause par les sciences de la nature pour leur manque de "netteté " épistémologique, leur incapacité à se donner un objet délimité et à produire des lois générales, en restant attachées à l'étude de singularités et de situations contextuelles (Mabilon-Bonfils, B. \& DeloryMomberger, C., dir., 2019, p. 10).

Il paraît pourtant fondamental d'analyser ces échecs et d'en tirer, dans une perspective constructive, un enseignement permettant de proposer des cadres éthiques appropriés 
aux recherches prenant appui sur les savoirs expérientiels de personnes en situation de vulnérabilité et visant leur empowerment, notamment lorsque ces recherches impliquent une collaboration avec des équipes s'inscrivant dans d'autres perspectives que celles des sciences de l'éducation et de la formation. Cette contribution, qui abordera les questions liées à la méthodologie de la recherche, sa mise en œuvre et le pilotage du projet s'inscrit dans cette intention.

7 Après avoir présenté la recherche, puis les perspectives épistémologiques et les principes méthodologiques dans lesquelles elle s'est inscrite, il sera rendu compte de l'évolution des choix, opérée au fil de la recherche pour tenter d'accéder à une compréhension et une restitution l'une et l'autre sensibles et respectueuses de l'expérience des participants, en dévoilant et analysant les écueils auxquels les équipes de recherche ont été confrontées.

\section{Présentation de la recherche}

8 La recherche sera présentée à travers les visées qu'elle poursuivait, les acteurs qu'elle mobilisait et le dispositif qui devait être mis en œuvre.

\subsection{Visées}

9 Un contrat engageait les membres des équipes de recherche vis-à-vis du commanditaire sur les objectifs.

10 La recherche visait principalement, en s'appuyant sur leur subjectivité (Thievenaz, Tourette-Turgis, 2015), à identifier et caractériser les handicaps et les ressources de personnes atteintes de deux maladies systémiques auto-immunes rares traitées par thérapie cellulaire ou par biothérapie - le lupus et la sclérodermie -, et ce, a priori, aux fins d'en améliorer la prise en charge et de contribuer à l'éducation thérapeutique des malades. Elle a pris appui sur la mise en récit du vécu des malades, initialement envisagée par le biais d'une e-plate-forme collaborative, dont il avait été espéré qu'elle serait favorable au partage et à la co-construction de savoirs, dans en "en-commun » entre les chercheurs et les malades "co-chercheurs", désignés ainsi car invités à adopter une posture de participants à la recherche. Il s'agissait de recueillir une parole adressée aux chercheurs, à l'institution de santé, aux autres malades, sur l'expérience de la vie avec une maladie et d'y entendre la singularité d'une construction individuelle en la mettant en relation avec le monde social.

11 Il s'agissait aussi, secondairement, de déterminer si la mise en place d'une e-plateforme collaborative, espace de partage numérique sur lequel les expériences des malades et des chercheurs seraient partagées, pouvait être favorable à cette coconstruction.

12 Enfin, il était attendu une évaluation de l'impact du dispositif de la recherche en termes d'empowerment ${ }^{1}$ en santé à travers l'évolution de la confiance en soi, du sentiment d'utilité sociale, de la mobilisation des capacités réflexives des participants. 


\subsection{Les acteurs}

13 La recherche mettait en relation: une équipe médicale spécialisée dans les maladies auto-immunes (équipe A), deux membres représentant de deux associations de malades, une quarantaine de malades «co-chercheurs» et une équipe de huit chercheurs en sciences de l'éducation et de la formation (équipe B), inscrits dans la perspective anthropologique de la recherche biographique en éducation, et pour certains, impliqués dans la mise en œuvre de dispositifs d'éducation thérapeutique et s'intéressant aux processus d'empowerment ${ }^{2}$ des malades.

L'équipe A avait en charge, en relation avec un chercheur de l'équipe B, l'inclusion des malades dans la recherche et l'organisation logistique: contact avec les malades, recueil des formulaires de consentement à la recherche, organisation des réunions du comité de suivi. Elle était par ailleurs gestionnaire de la recherche. Les représentants des associations participaient également à l'inclusion des malades en diffusant un appel à participation. Ils avaient vocation à représenter les malades dans les instances décisionnelles. L'équipe $\mathrm{B}$ avaient la responsabilité de la construction des récits des cochercheurs et de leurs interprétations. Un comité de suivi réunissant des membres de chaque équipe se tenait mensuellement pour s'informer des avancées de la production des «données» et prendre des décisions face aux difficultés rencontrées. Y participaient deux des chercheurs de l'équipe B - l'un responsable de la liaison entre les co-chercheurs et les chercheurs, et l'autre responsable de la coordination des chercheurs et des relations avec l'équipe médicale -, un ingénieur de recherche appartenant à l'équipe médicale, une infirmière et le professeur responsable du service concerné, ainsi que les représentants des associations de malades.

Le groupe des co-chercheurs, hommes et femmes d'âges différents, a finalement réuni 18 personnes atteintes de sclérodermie et 10 de lupus. Leurs maladies pouvant affecter plusieurs organes et détruire leurs propres cellules, provoquant des difficultés cumulatives lourdement handicapantes et pouvant conduire au décès, tous les malades concernés par la recherche bénéficiaient de traitements innovants visant à améliorer leur qualité de vie : biothérapie et thérapie cellulaire par greffe de cellules souches.

\subsection{Dispositif}

16 La recherche débutait avec la phase d'inclusion des malades au dispositif de recherche. Pour les malades atteints de sclérodermie, ils se trouvaient informés de la recherche par un membre de l'équipe $A$ et un chercheur de l'équipe $B$ lors d'une de leurs hospitalisations. Les malades atteints de lupus, dispersés sur le territoire français, premièrement informés par leurs médecins, étaient contactés par téléphone et sollicités pour participer à la recherche. Aux uns et aux autres, les modalités de mise en œuvre et les objectifs de la recherche étaient présentées dans les détails et leur statut de co-chercheur explicité.

17 La recherche devait se développer au cœur d'une communauté virtuelle et protégée avec pour support la e-plateforme collaborative Dropbox. Celle-ci avait été retenue car il n'était pas possible, entre autres pour des questions de confidentialité et de droit d'accès, de recourir aux plates-formes numériques des universités engagées dans la recherche et qui proposaient des outils collaboratifs plus simples à utiliser. Il a donc été choisi un outil indépendant, sur lequel chaque chercheur et chaque malade co- 
chercheur pouvait être inscrit par le responsable de la plate-forme. La plate-forme disposait de plusieurs espaces : un espace privé de communication entre les chercheurs de l'équipe $\mathrm{B}$, un espace d'information partagé par tous sur les modalités et les étapes de la recherche, un espace privé par binôme de chercheur et co-chercheur, et un espace de publication partagé par les co-chercheurs et les chercheurs de l'équipe B. L'équipe $\mathrm{A}$ et les représentants d'associations de malades n'avaient pas d'accès à cette plate-forme. Après obtention de l'accord des malades, une première formation aux outils numériques et à l'usage de la plate-forme leur était délivrée, en présence à l'hôpital pour les malades atteints de sclérodermie, au téléphone pour ceux atteints de lupus, par le chercheur de l'équipe A responsable de la liaison entre les co-chercheurs et les chercheurs.

19 L'équipe B avait ensuite la responsabilité d'accompagner les co-chercheurs dans la construction d'un récit de leur expérience. Les participants étaient invités à retracer leur parcours de vie, à décrire et à analyser l'impact de la maladie sur leurs conditions de vie, les adaptations et les stratégies mises en œuvre pour lui faire face, l'évolution de leur parcours et de leur développement personnel dans leur vie " avec la maladie ».

Cette étape de construction du récit reposait sur une collaboration en binômes entre un chercheur et un co-chercheur, sur leur espace exclusif et privé de la plate-forme permettant de respecter la confidentialité des données et l'anonymat (cf. annexe "Méthode pour le recueil des données »). Le co-chercheur devait se trouver libre de sa parole et de ses modalités d'expression et le chercheur l'accompagnait avec sa singularité, en construisant avec lui une relation de confiance et l'invitant progressivement à raconter son expérience.

21 Il était par ailleurs attendu par l'équipe A que soit renseigné un test psychologique de type «QCM» concernant l'estime d'eux-mêmes des co-chercheurs (échelle de Rosenberg). Soumis au début et à la fin de la recherche, ce test devait permettre d'en mesurer les effets sur les participants. Pour les membres de l'équipe A, s'appuyant sur leurs expériences de recherches menées dans des perspectives biomédicales, l'utilisation de questionnaires comparatifs s'avérait nécessaire pour garantir la scientificité d'une recherche. Cette demande qui laissait présager des difficultés à venir, intervenant dans une période d'ajustement entre les équipes a fait l'objet de réserves de la part de l'équipe B du fait de la perspective qualitative adoptée et du nombre de participants. Pourtant elle ne s'y est pas opposé catégoriquement, par crainte que la recherche soit abandonnée.

L'ensemble des récits, les interactions entre tous les acteurs et les questionnaires devaient constituer le matériel à interpréter pour produire les connaissances attendues, dans la perspective épistémologique et méthodologique de la recherche biographique en éducation.

\section{Perspectives épistémologiques et méthodologiques}

Les perspectives épistémologiques et méthodologiques de la recherche mise en œuvre seront abordées dans leurs interactions en matière de production et d'interprétation de données biographiques. 


\subsection{Production des récits}

24 êtres humains donnent une forme et un sens à leurs expériences, en particulier en racontant et en "écrivant» (en biographiant) leur existence. Elle donne à voir la configuration « que chacun donne à sa propre existence et qui fonde le sentiment qu'il a de lui-même " (Delory-Momberger, 2014, p. 79). Plus précisément, elle observe comment les sujets inscrivent leur existence dans des schémas temporels orientés dont les récits forment la trame, s'appuyant ainsi sur la capacité qu'ils ont d'« agir » sur leurs représentations et d'entrer dans des processus d'apprentissage, de construction d'eux-mêmes et de leurs expériences.

recherche biographique considère que le chercheur peut comprendre les expériences que relatent les participants à la recherche parce qu'il est lui-même un être humain, relié à l'ensemble des êtres humains, grâce aux histoires que chacun se raconte et avec lesquelles il "interprète" les événements de sa vie. Cette compréhension herméneutique a une dimension individuelle autant que sociale : au niveau individuel, nous mettons en relation et structurons des événements de notre vie pour en faire une histoire, "notre» histoire, que nous modifions au fil du temps, la réinterprétant à la lueur de nouvelles expériences, de nouveaux apprentissages; au niveau social, nous appartenons tous à un système social, et chacune de nos pratiques s'inscrit dans une relation avec ce système et en dit quelque chose. Ainsi que l'indique Ferrarotti, la «subjectivité inhérente à l'autobiographie» peut devenir une connaissance scientifique dans la mesure où «chaque narration autobiographique raconte, selon une coupe horizontale ou verticale, une pratique humaine. Or [...] toute pratique individuelle humaine est une activité synthétique, une totalisation active de tout le système social» (Ferrarotti, 2013, p. 52). La recherche biographique envisage ainsi chaque récit singulier comme pouvant contribuer à produire des connaissances sur ce système social sans risquer d'effacer la personne dans une perspective homogénéisante et globalisante.

Une telle approche implique d'essayer de sortir du rapport hiérarchique induit par la situation de recherche pour constituer un espace, le plus horizontal possible, de rencontre et de construction partagée de connaissances entre la personne du chercheur et la personne envisagée comme co-chercheure et donc comme interlocutrice capable de mobiliser des savoirs à partir de son expérience et de les transmettre. Dans cet espace du sensible et de l'intersubjectivité, le vécu raconté et les émotions qui les accompagnent peuvent être accueillis et interprétés par le chercheur qui développe une attitude empathique. Celle-ci relève « d'un processus dynamique qui s'origine dans la rencontre de consciences incarnées, laquelle se réalise sur fond d'ouverture à autrui, vu comme son alter ego, son égal » (Janner-Raimondi, 2017, p. 54). Elle s'appuie sur un véritable déplacement du chercheur permettant, à partir de sa propre expérience, d'accéder à une compréhension herméneutique du sujet. Elle engage donc la responsabilité éthique du chercheur et exclut toute perspective utilitariste vis-à-vis des participants.

Pour accompagner les malades dans le développement de leur pouvoir d'agir, rejoignant en cela la perspective proposée par Chauvier (2012), il s'agissait donc de construire un dispositif de recherche dans lequel les participants ne se trouvent pas en position de simples «informateurs ». Ils étaient invités, plutôt qu'à être uniquement 
«impliqués» dans la recherche (Ardoino, 1992), à s'y engager pleinement ${ }^{3}$. Les cochercheurs se voyaient donc proposer de réagir à la lecture des récits des autres malades et des interprétations proposées par les chercheurs, participant donc à leur élaboration. Il était également prévu qu'ils puissent participer à la diffusion des résultats de la recherche, en s'exprimant sur leur vécu lors de présentations de la recherche dans des colloques ${ }^{4}$.

Les co-chercheurs, en effectuant un travail de mise en sens et en forme de leurs expériences, «enquêtaient »sur eux-mêmes, en même temps que les chercheurs devaient chercher à comprendre le travail qu'ils effectuaient dans le cadre de la mise en récit de ces expériences. L'objectif visé était ainsi d'ouvrir un espace de coproduction, de transmission et d'échanges de savoirs pour recueillir une parole située sur l'expérience de la maladie et y entendre la singularité d'une construction individuelle dans sa relation avec les autres et avec le monde social. Il devait être possible d'y saisir comment chacun conjuguait son expérience et les mondes communs de penser et d'agir dans lesquels il se trouvait engagé, afin de compléter les perspectives biomédicales, dans le but d'améliorer les soins. Prendre appui sur l'expérience des malades co-chercheurs dans la gestion quotidienne de leur maladie et de leurs traitements pouvait en effet ouvrir un accès à une meilleure compréhension de certains aspects de leur parcours de vie et de soin : notamment à la manière dont ils géraient quotidiennement leur maladie et leurs traitements, les stratégies adoptées, inventées, pour poursuivre leur existence mais aussi leurs ressentis. Le souci de leur empowerment impliquait qu'ils ne soient pas réduits à leur statut de malade, mais considérés dans la singularité du parcours global de leur personne et dans le respect de leur autonomie. Les interprétations des chercheurs s'attachaient à restituer ce parcours.

\subsection{Interprétations}

Dans la perspective herméneutique de la recherche biographique en éducation, les données de la recherche, ici constituées des récits produits par les malades cochercheurs et des entretiens réalisés avec eux sur le vécu de la recherche, font l'objet d'une interprétation. Celle-ci prend appui sur une trame constituée de quatre catégories d'analyse, adaptée par Delory-Momberger (2014, pp. 89-92) à partir des travaux de Heinz (2000, pp. 165-186). Elle amène à observer :

- les formes du discours (narratif, évaluatif, explicatif, descriptif), qui témoignent de la mise en réflexivité du co-chercheur ;

- les schémas d'action/les systèmes d'acteurs, qui témoignent du rapport aux situations et aux événements (actif, passif, stratège, attentiste...) ;

- les thèmes récurrents qui organisent l'action et qui sont des clefs d'interprétation du vécu ;

- la gestion biographique des thèmes : c'est-à-dire la manière dont les narrateurs apprécient, négocient, ajustent leur action et la réalité socio-individuelle. Il s'agit dans cette catégorie d'observer d'éventuelles confrontations entre la biographie d'expérience du malade et les biographies sociales standardisées des mondes sociaux auxquels il appartient. Les enjeux politiques de ces confrontations apparaissent dans ce qu'elles peuvent manifester du pouvoir d'agir de celui qui produit son récit.

30 Ces quatre catégories, qu'il s'agit de faire entrer en relation les unes avec les autres, ont pour objectif de permettre d'observer comment le narrateur se «fabrique» dans le 
récit, comment il interagit avec son environnement, et en l'occurrence dans cette recherche, comment il vit et éprouve sa maladie dans la relation à soi, à la société, aux proches et à son entourage, aux soignants et au soin.

Si la méthode d'interprétation a pu être mise en œuvre telle qu'elle avait été envisagée, de nombreux ajustements ont été nécessaires pour tenter de dépasser des difficultés rencontrées dès le début de la recherche.

\section{Difficultés rencontrées et ajustements méthodologiques}

32 En effet, certains écarts décalages entre le dispositif envisagé et sa mise en œuvre effective se sont avérés nécessaires pour tenter d'atteindre les objectifs de la recherche. Rendre visible ce «bricolage » méthodologique semble pertinent pour mettre au jour certains enjeux et écueils de recherches visant à accéder à l'expérience sensible de personnes en situation de vulnérabilité. Les difficultés sont apparues dès le début de la recherche en matière d'inclusion des malades, d'utilisation de la plate-forme numérique, de prise en compte de la singularité des co-chercheurs, mais aussi de celle des chercheurs, d'usage de questionnaires, interrogeant aussi bien les objectifs, les enjeux et les modalités de recherche.

\subsection{L'inclusion dans la recherche}

La première difficulté rencontrée concerne l'inclusion des malades à la recherche.

À l'échéance de la phase prévue d'inclusion, seuls 18 malades avaient accepté de participer à la recherche. L'équipe $\mathrm{A}$ a donc souhaité que les inclusions à la recherche puissent être progressives et que d'autres malades puissent rejoindre la recherche en cours de route. Les chercheurs de l'équipe B ont dû adapter le dispositif pour accueillir progressivement les co-chercheurs et démarrer avec eux de manière différenciée les phases d'écriture, renonçant au dépôt simultané sur l'espace public de la plate-forme des productions correspondant aux différentes phases d'écriture. Cette situation a rendu plus difficile la construction d'une cohésion dans l'équipe des co-chercheurs.

Le comité de suivi de la recherche a choisi de s'appuyer sur les éléments transversaux du vécu des deux maladies concernées pour poursuivre la recherche avec les 28 cochercheurs finalement inclus, malgré l'asymétrie entre les porteurs de lupus et les porteurs de sclérodermie. Il s'est en effet avéré difficile d'inclure des malades souffrant de lupus et, finalement, la recherche a concerné 20 personnes souffrant de sclérodermie systémique - pour la plupart soignées par thérapies innovantes dans le même hôpital référent auquel appartenaient les membres de l'équipe A partie prenante de la recherche - et 8 personnes souffrant de lupus, dispersées sur le territoire français. Il semble que l'influence des membres de l'équipe A impliquée ait eu un rôle sur le désir d'investissement de malades. S'il est possible que les informations y aient été plus clairement diffusées, les entretiens ont aussi révélé que certains malades ont accepté de participer pour satisfaire à la demande de leur médecin. Même si le spécialiste, en proposant la recherche à ses patients, spécifiait qu'il sortait de son rôle habituel, il restait néanmoins la personne entre les mains de laquelle reposaient bien souvent les espoirs de retour à un meilleur état de santé ou qui avait réussi à améliorer leurs 
conditions de vie. Les co-chercheurs ont bien souvent fait état de leur admiration à son égard et leur consentement à la recherche avait souvent en premier lieu été donné pour le remercier. Ce consentement pouvait inscrire les co-chercheurs dans la figure $\mathrm{du}$ «bon malade » qui mérite d'être «bien » soigné mise en évidence par Ogien (2017). Il a $\mathrm{pu}$ être recueilli indépendamment d'un réel intérêt ou d'une compréhension des objectifs de la recherche. L'attitude de certains co-chercheurs témoignait d'un souci de «bien » faire, parfois source de stress pour eux. Ces circonstances contribuent déjà en elles-mêmes à questionner le respect de l'initiative et de l'autonomie des malades inclus dans la recherche.

\subsection{La plate-forme}

La seconde difficulté rencontrée tient à l'utilisation de la plate-forme sur laquelle le malade co-chercheur devait déposer les différentes étapes du récit réalisé avec l'accompagnement d'un chercheur. Finalement, seuls 19 co-chercheurs sur 28 ont utilisé la plate-forme Dropbox.

Cette difficulté tient en partie à des problèmes d'ordres :

- techniques : non-maitrise de l'outil informatique, absence de connexion Internet, défaut de matériel...

- médicaux : handicap physique lié aux maladies concernées qui empêche de mobiliser les doigts, fatigue

- culturels : accès parfois difficile aux pratiques d'écriture

Pour remédier à ces difficultés, le choix a été fait d'établir des contacts téléphoniques pour guider les co-chercheurs dans l'utilisation de Dropbox. Cela n'a pas toujours suffi et la personne responsable des relations entre chercheurs et co-chercheurs a proposé, pour remédier à la situation, des échanges par l'intermédiaire d'une boîte email dédiée permettant de préserver l'anonymat de ces derniers.

40 Finalement, les discussions entre les chercheurs de l'équipe B et les co-chercheurs faisant état pour la majorité d'entre ceux-ci d'un besoin d'établir un contact direct pour instaurer une relation de confiance, des entretiens téléphoniques ou visiophoniques enregistrés ou des entretiens en présence lors de leurs hospitalisations (notamment pour les malades atteints de sclérodermie systémique) leur ont été proposés. Il apparaissait nécessaire qu'ils puissent nouer une relation de personne à personne, en toute confiance et confidentialité, avec les chercheurs de l'équipe B, sortant d'une relation au personnel médical en charge de leurs soins médicaux pouvant être perçue comme réductrice dans la mesure où elle les envisageait essentiellement dans leur dimension biomédicale.

41 Cette disposition nouvelle impliquait une attention particulière à l'anonymat des écrits déposés sur la plate-forme, à laquelle l'équipe A n'avait pas accès car elle connaissait parfois trop bien les participants pour ne pas les identifier malgré les pseudonymes.

La proposition d'entretiens changeait profondément les modalités de relation entre chercheurs et co-chercheurs prévues par la recherche, introduisant une dimension physique dans la rencontre (voix, corps), qui n'avait pas été envisagée au début de la recherche. La relation établie, par la voix ou la présence, liait les chercheurs aux cochercheurs autrement que par l'intermédiaire d'une plate-forme, donnant accès de 
façon plus sensible aux émotions et aux souffrances, et par conséquent à la vulnérabilité de ces derniers.

Cette modalité se constituait comme la seule véritable possibilité de partager l'expérience subjective de la recherche entre le chercheur et le co-chercheur, mais elle freinait la dynamique de co-construction de savoirs. Le manque d'interactions sur la plate-forme limitait les échanges entre les co-chercheurs, qui s'installaient dans une relation duelle avec le chercheur, moins propice à limiter la situation asymétrique de la recherche que la relation collective envisagée premièrement, reposant sur une dynamique de groupe.

\subsection{La singularité des co-chercheurs}

Tous les co-chercheurs n'ont pas pu ou voulu respecter les phases d'écriture telles qu'elles étaient envisagées. La modélisation des récits qui découlait de la chronologie et des axes narratifs envisagés est apparue trop rigide à certains d'entre eux. Par ailleurs, la temporalité prévue de la recherche ne rencontrait pas toujours la leur. Ils ne pouvaient pas tous respecter les délais prévus, en fonction de leur disponibilité, de leur accès au numérique (ordinateur ou connexion parfois défaillante), mais aussi de leur état émotionnel, affecté par la mise en récit de leur expérience.

L'état de santé fluctuant de certains participants a également posé question. Il s'agissait de ne pas leur imposer un rythme qui les fatiguerait davantage, ou, au contraire, de pouvoir répondre au besoin qu'ils avaient d'avancer plus vite. Certains souhaitaient parfois finir leur récit avant une hospitalisation qui les rendrait indisponibles ou en fonction d'événements de leur vie qui allaient leur demander du temps.

Il a été choisi de s'adapter et de permettre aux co-chercheurs de produire leurs récits selon les modalités qui leur convenaient, en fonction de leurs possibilités et de leurs besoins. Certains co-chercheurs n'ont pas pu (décès prématuré) ou voulu donner une autorisation de partager leurs récits et ils ont été respectés. Un des participants, en fin de vie, a pu compléter l'ensemble de son récit dont il avait rédigé la première phase par écrit, lors d'un entretien à l'hôpital, et choisir de le diffuser vers les autres cochercheurs et chercheurs avant son décès. Deux co-chercheurs ont fait le choix de se retirer de la recherche qui leur demandait un trop gros investissement au regard de leurs activités, dont celles liées à leurs soins.

Par ailleurs, si la plupart des co-chercheurs ont accepté de lire les interprétations proposées par les chercheurs, peu les ont contestées ou modifiées. Il est apparu évident qu'ils s'en remettaient à l'expertise des chercheurs, avec lesquels s'étaient noués des liens de confiance, mais dont la position légitimait de toute façon pour nombre de cochercheurs les interprétations proposées.

\subsection{La singularité des chercheurs en sciences de l'éducation et de la formation}

Une autre difficulté rencontrée a été la mise à l'épreuve de la subjectivité des chercheurs, toujours singulière. Il n'est en effet pas anodin de se confronter à la souffrance d'autrui, qui renvoie à la responsabilité prise en tant que professionnel, mais aussi en tant qu'humain, face à la parole sollicitée et celui qui l'énonce. Mais qui renvoie aussi à son propre vécu, créant parfois du trouble dans l'interprétation qui en 
était faite. La mise en sens de leurs expériences par les co-chercheurs pouvait amener à une déstabilisation du sens que le chercheur donnait jusque-là à ses propres expériences de la maladie et de la santé. Les réunions mensuelles de l'équipe B se sont constituées en espace de prise de recul où consolider la posture empathique adoptée, accueillir et mettre au travail les émotions rencontrées, pour proposer une interprétation permettant de rendre le sensible des expériences livrées par les cochercheurs, nécessairement traversées de l'expérience de la relation instaurée avec les chercheurs. L'enjeu de la recherche ne se limitait pas à la production de connaissances scientifiques sur les effets de certains traitements ou à développer des pratiques d'éducation thérapeutique permettant d'acquérir des compétences pour mieux gérer la vie avec une sclérodermie ou un lupus systémique. Pour les co-chercheurs, il s'agissait de rendre visible leur expérience sensible, grâce aux publications et communications qui devaient suivre la recherche.

\subsection{Le questionnaire sur l'estime de soi}

Il s'est avéré très difficile de proposer aux co-chercheurs le test sur l'estime de soi retenu par l'équipe A dans le projet de la recherche. Il était difficile de le proposer en début de recherche en raison de l'inclusion échelonnée des participants et de l'obstacle qu'il constituait à la construction d'une relation de confiance. À la fin de la recherche, compte-tenu du nombre limité de participants, son intérêt pouvait aussi être mis en doute. Mais par ailleurs, la démarche entrait en contradiction avec les principes non directifs de la recherche biographique en éducation et les objectifs qualitatifs de la recherche. L'équipe $\mathrm{A}$ a eu du mal à entendre ces arguments, remettant en cause la scientificité des connaissances produites indépendamment de questionnaires et a exigé qu'ils fassent l'objet d'une passation, pour respecter la méthodologie envisagée au début de la recherche. Ils ont donc été proposés par les chercheurs de l'équipe $B$, dans le cadre de l'entretien bilan sur le vécu de la recherche, qui ouvrait la possibilité de s'exprimer en dehors et au-delà du cadre du test. Malgré les précautions prises, certaines questions ont constitué pour certains co-chercheurs une véritable violence (face auxquelles ils ont exprimé colère ou souffrance).

\subsection{Les objectifs, les enjeux et les modalités de recherche}

50 La question épineuse du test sur l'estime de soi était révélatrice de dissensions plus profondes entre l'équipe $\mathrm{B}$ et l'équipe $\mathrm{A}$, liées à la spécificité des regards portés sur les objectifs, les enjeux et les modalités de la recherche. Cette différence d'approche entrait en relation avec un objectif resté longtemps implicite dans le projet et le contrat de recherche et dont l'équipe B n'avait pas eu conscience : l'équipe A escomptait que la recherche mettrait en évidence l'amélioration de la qualité de vie des patients grâce à l'utilisation de traitements innovants et c'est ce qui avait motivé principalement sa participation à un projet répondant à l'appel du commanditaire. Mais l'équipe $B$ défendait pour sa part la légitimité de l'approche de la recherche biographique en éducation dans le domaine de la santé. Ainsi l'ensemble de la recherche reposait-il sur des malentendus conséquents qui n'ont été mis à jour que trop tardivement.

Les récits d'expérience recueillis et les interactions entre les chercheurs et les cochercheurs ont permis de construire des connaissances sur les situations 
handicapantes rencontrées par ces derniers et les ressources éventuellement déployées pour les dépasser. Ces récits et ces témoignages mettaient aussi en évidence l'amélioration de la qualité de vie et de l'estime d'eux-mêmes des co-chercheurs après certaines thérapies, mais sans permettre de fournir "les données statistiques" réclamées par l'équipe $\mathrm{A}$, qui souhaitait, avant toute autre publication dans une revue de sciences humaines, produire un article princeps, original et inédit, s'inscrivant dans la tradition et la perspective biomédicale. Cette publication devait mettre en évidence les bienfaits des thérapies innovantes. Il n'a pas été possible de faire entendre à l'équipe A que l'exercice était impossible et un lourd travail de rédaction a été effectué par le comité de pilotage pour tenter de satisfaire malgré tout à ces exigences et répondre aux critères d'une revue scientifique tournée vers les professionnels de la santé publique qui n'a pas accepté la publication. Cet échec n'a pas débouché sur une autorisation de se tourner vers des revues de sciences humaines et sociales qui auraient accueilli sans difficulté des articles sur les différentes problématiques liées à la recherche. Le contrat signé par les chercheurs spécifiait que toute publication liée à la recherche devait faire l'objet d'un consensus des membres du comité de suivi de la recherche et ce, sans délai de prescription. Les publications en sciences humaines n'ayant pas été autorisées, il n'a donc été accordé aucune considération aux connaissances produites sur les situations de handicaps rencontrées par les malades, aux ressources développées pour y faire face et à la parole que les malades co-chercheurs avaient déployée et souhaitaient partager. Si des propositions intéressantes en matière d'éducation thérapeutique ont pu émerger des interprétations de la recherche, la rupture de communication ne permet pas à l'équipe B d'avoir connaissance de leur mise en œuvre. Le constat global est donc celui d'un échec.

La perspective déductive dans laquelle l'équipe A s'inscrivait n'a pas pu rencontrer la perspective compréhensive de la recherche biographique en éducation, s'appuyant sur une démarche inductive de nature à favoriser le développement du pouvoir d'agir des participants, et ce, même en partageant la même finalité : le mieux-être des malades.

\section{Conclusion}

Cette contribution a voulu faire état d'une recherche dont les conclusions n'ont pas pu être diffusées mais qui met en évidence un certain nombre d'enjeux en matière de recherche qu'il semble important de mettre au jour pour construire un cadre éthique permettant de faire entendre la voix des personnes «invisibilisées» sans exercer de violence symbolique à leur encontre.

54 La tentation est forte de participer à des recherches collaboratives mêlant chercheurs en sciences de l'éducation et de la formation et équipes médicales, nourrie par l'espoir de faire évoluer certaines postures descendantes mises en cause par nombre de chercheurs mais aussi d'associations de malades. Placer les participants en position d'interlocuteurs pour construire des savoirs complémentaires des perspectives biomédicales présente un intérêt incontestable.

Mais il s'agit d'élucider au préalable les enjeux de ces recherches pour les différents acteurs qu'elles réunissent. Il serait naif de ne pas envisager que les équipes médicales puissent avoir besoin d'instrumentaliser ces savoirs pour renforcer leurs prérogatives, ou que les équipes de chercheurs en sciences de l'éducation et de la formation en attendent de faire entendre leur position dans le champ scientifique, cela posant 
éthiquement problème si les participants co-chercheurs ne restent pas les premiers bénéficiaires de leur engagement dans la recherche.

Il s'agit également d'analyser rigoureusement les conditions structurelles et contextuelles de la recherche, qui définissent les potentialités du changement social espéré dans les recherches visant un empowerment des acteurs. La perspective de la recherche biographique favorise manifestement la construction de conditions individuelles à ce changement, entre autres, par le développement d'une démarche conscientisante, mais l'empowerment ne devient effectif que si les conditions structurelles le permettent, comme le défend Le Bossé (2003, pp. 34-36) qui considère que la prise en compte simultanée des conditions structurelles et individuelles du changement social et la prise en compte des contextes d'application sont deux des composantes nécessaires à l'empowerment.

Dans le cas de la recherche relatée, certains objectifs n'avaient pas été suffisamment explicités pour faire apparaître des incompatibilités qui auraient préservé les participants co-chercheurs d'un investissement considérable dont le profit n'a été que partiel. Si la recherche a pour eux été l'occasion d'un partage de ressources, d'une élaboration du sens de leur parcours parfois propice au développement de leur pouvoir d'agir (certains se sont engagés dans des associations de malades, ont repris des formations...), l'absence de socialisation de leurs productions et des interprétations qui en ont été faites en a limité considérablement les effets en matière d'empowerment. Les enjeux de reconnaissance liés à la recherche impliquaient que la mise en récit des expériences puisse sortir d'un cercle privé. Le regard de l'autre permet de vérifier que l'on détient un savoir, la légitimité de ce savoir provenant de son partage dans un groupe d'appartenance où la parole de chacun est reçue et entendue en relation avec celle des autres. Mais le récit de soi adressé à un autre ou partagé dans un groupe d'appartenance, qui nourrit la confiance en soi, reste un point de départ, comme Caillaux le met en évidence (2010). Les relations interpersonnelles ne sont pas suffisantes pour nourrir une estime de soi favorisant le développement d'un empowerment. Elles doivent être dépassées par l'inscription de la parole «dans des espaces où elle trouve une dimension collective et contribue à l'ensemble social (Honneth, 2000, p. 147), ce que la publication ou la participation à des colloques avec les chercheurs aurait rendu possible.

Au final, cette recherche que l'équipe de sciences de l'éducation et de la formation a abordée dans la perspective d'une déhiérarchisation des savoirs témoigne d'une part de la difficulté à véritablement co-construire des connaissances dans un regroupement pluridisciplinaire d'équipes dont les objectifs peuvent être différents et, d'autre part, de la difficulté à constituer les savoirs expérientiels en outils de renversement de certains rapports de pouvoir.

59 Mettre les participants en situation d'interlocution implique de socialiser leur parole dans l'espace public. La volonté d'inscrire les relations avec les participants à une recherche dans une symétrie ne suffit pas et doit se trouver dépassée, pour pouvoir proposer un véritable statut de co-chercheur, par une réelle inclusion aux instances de décision de la recherche. Dans notre cas, cette volonté semblait pouvoir être assurée par la présence dans le comité de suivi de deux représentants d'une association de malades. Mais celle-ci n'assurait pas leur représentativité. En effet, il s'avère que ces deux représentants, souffrant l'un et l'autre d'une des maladies concernées par la recherche et dont l'un se trouvait être médecin spécialiste, s'inscrivaient dans une 
proximité de l'équipe médicale gestionnaire de la recherche et partageaient sa position concernant les thérapies mises en œuvre.

Les participants ne disposaient donc d'aucun pouvoir décisionnel sur le devenir des connaissances produites Faire entrer les participants à une recherche dans les instances décisionnelles n'est pas sans poser de difficultés, notamment dans des recherches amenant à dialoguer avec un nombre important de participants. Mais il paraît évident que, sans réel pouvoir des participants co-chercheurs sur le pilotage de la recherche, la symétrie de position que laisse entendre le statut de co-chercheur s'avère trompeuse. Elle peut même se trouver contre-productive et renforcer une objectivation des participants peu respectueuse de la singularité de leur expérience. Quand les recherches ne permettent pas de garantir aux participants une véritable position de co-chercheurs, garante d'un plein statut d'auteur, participant à leur empowerment et par là-même au renversement de rapports de domination qui produisent l'assujettissement à un ordre social, culturel ou, comme dans notre cas, biomédical, il paraît préférable de ne pas adopter cette dénomination et, comme le propose Jean-Yves Rochex, d'éviter «les positions et affirmations relevant du brouillage épistémologique » qui ne « sauraient [...] être une arme efficace pour réparer ou combattre les processus de domination, de disqualification et d'injustice sociale " (Rochex, 2010, p.11), mais plutôt «de reconnaître la spécificité, l'efficacité et la pertinence de chaque type ou genre d'activité sociale dans son ordre propre » (Ibid) $)^{5}$, le travail de co-élaboration de sens mené en situation par le chercheur et les participants se différenciant du travail d'élaboration théorique effectué par le chercheur d'autre part.

\section{BIBLIOGRAPHIE}

Ardoino, J. (1992). L'implication. Lyon : Voies Livres.

Bassleer, B., Boulanger, J-M., Menozzi, Ch. (2016). L'empowerment des patients : Pourquoi et comment. Liège : $\mathrm{CHU}$ de Liège.

Baeza, C. (2019). Savoirs sensibles. In C. Delory-Momberger (dir.). Vocabulaire des histoires et de la recherche biographique en éducation. Toulouse : Érès.

Caillaux, M. (2011). Comme des orpailleurs : de la misère à la pauvreté, les relations comme chemin de libération. Paris : L'Harmattan.

Chauvier, E. (2012). Anthropologie de l'ordinaire. Pour retrouver la voix des déclassés. Journal des anthropologues, 128-129, 209-221.

Delory-Momberger, C. (2014). De la recherche biographique en éducation. Fondements, méthodes, pratiques. Paris : Téraèdre.

Ferrarotti, F. (2013). Histoires et histoires de vie. Paris : Téraèdre.

Flora, L. (2011). Acteur, auteur de sa santé jusqu'au dernier souffle. Revue Générale de Droit Médical, $38,239-253$. 
Honneth, A. (2000). La lutte pour la reconnaissance. Paris : Cerf.

Janner-Raimondi, M. (2017). Visages de l'empathie en éducation. Nîmes : Champ social.

Jouet, E., Flora, L., Las Vergnas, O. (2010). Construction et reconnaissance des savoirs expérientiels des patients. Pratiques de formation - Analyse, 58/59, 13-94.

Le Bossé, Y. (2003). De l'« habilitation » au « pouvoir d'agir » : vers une appréhension plus circonscrite de la notion d'empowerment. Nouvelles pratiques sociales, 16 (2), 30-51. https://doi.org/ 10.7202/009841ar

Mazereau, P. (2016). L'élargissement de la coopération aux usagers et professionnels non spécialisés : une nécessité dans l'accompagnement des parcours des personnes en situation de handicap. Forum, 148, 60-65.

Mabilon-Bonfils, B., Delory-Momberger, C. (2019). (dir.). À quoi servent les sciences de l'éducation? Paris : ESF.

Ogien, O. (2017). Mes mille et une nuits. La maladie comme drame et comme comédie. Paris : Albin Michel.

Rhéaume, J. (2019). Pouvoir d'agir / empowerment. In C. Delory-Momberger (dir.). Vocabulaire des histoires et de la recherche biographique en éducation (p. 127-129). Toulouse : Érès.

Rochex, J.-Y. (2010). Approches cliniques et recherche en éducation, Recherche et formation, 65. [en ligne] http://journals.openedition.org/rechercheformation/165

Thievenaz J., Tourette-Turgis, C. (2015). Penser l'expérience du soin et de la maladie. Une approche par l'activité. Bruxelles : De Boeck.

\section{ANNEXES}

\section{Annexe : Méthode pour le recueil du matériau biographique}

\section{Organisation :}

\section{- Écriture}

Une fois formés par le responsable des relations chercheurs-co-chercheurs aux outils numériques nécessaires à la recherche (Dropbox et skype), l'interaction avec les chercheurs durera environ 6 mois.

Une première semaine sera consacrée à des échanges permettant de faire connaissance avec le chercheur avec qui le participant forme un binôme en tant que cochercheur et de mettre en place avec lui les modalités de la collaboration.

Durant le reste de ces 6 mois se succèderont 4 phases d'écriture de récits, organisés autour de 4 thèmes d'écriture correspondant aux questions principales suivantes.

Phase 1 : Racontez comment tout cela a commencé... votre santé.

Phase 2 : Qu'est-ce qui vous a aidé (e) ? Qu'est-ce qui vous a permis de faire face ?

Phase 3 : Qu'est-ce que cela vous a appris?

Phase 4 : Qu'est-ce que vous aimeriez transmettre à d'autres et comment aimeriez-vous le faire? 
Pour chacune des phases de récit, une série de sous-questions qui viendront relayer, expliciter, détailler seulement quand nécessaire l'amorce principale et faciliter l'avancée et la construction du récit. Élaborées par le groupe de chercheurs en sciences de l'éducation et de la formation, elles seront ajustées dans le suivi de chaque nouvelle séquence narrative lors des réunions de synthèse qui viendront ponctuer le déroulement de cette partie de la recherche (recueil des récits) :

Phase 1 : Comment cela s'est passé ? L'annonce ? La manifestation ? Le sentiment que l'on a (mental, psychique, affectif) ? Le rapport à l'entourage familial, entourage professionnel?

Phase 2 : Qu'est-ce qui vous a aidé (e) ? Quoi ? Quel traitement ? Quels dispositifs ? Qui ? Quelles rencontres? Qu'est-ce qui vous a permis de faire face? Qu'est-ce que vous avez mis en place pour faire face ? Comment avez-vous évolué avec cela ? Qu'est-ce qu'il faut changer et améliorer par rapport à ce qui ne va pas dans cette maladie ou les manières de la prendre en charge?

Phase 3 : Qu'est-ce que cela vous a appris ? Qu'est-ce que vous avez appris sur vousmême et sur vos ressources personnelles? Qu'est-ce que vous avez dû apprendre à faire que vous n'auriez pas eu besoin d'apprendre si vous n'aviez pas été malade ? Racontezmoi comment vous avez appris cela? Qu'est-ce que vous avez appris dans le domaine des réactions de votre corps aux symptômes de votre maladie ? Les réactions de votre corps aux traitements? Comment vous avez appris à repousser les limites ? À prendre des risques, à vous protéger ? Comment avez-vous appris à vous situer/défendre/ protéger dans le domaine de la vie sociale et professionnelle?

Phase 4 : Qu'est-ce que vous aimeriez transmettre à d'autres et comment aimeriez-vous le faire ? Une technicité ? Une expérience ? Un savoir de la relation? Des stratégies dans la vie sociale et professionnelle ? Des modalités dans la transmission (présentiel, numérique, associatif, réseau, etc.) ?

\section{- Échanges}

À la fin de chaque thème d'écriture, les participants choisiront dans leur écrit ce qu'ils acceptent de publier, ce qui pourra être lu par l'ensemble des chercheurs et des cochercheurs et ils pourront aussi lire l'expérience des autres. Un espace collaboratif (dossier travaux collaboratifs) en ligne permettra de partager les écrits et d'échanger avec les autres co-chercheurs.

\section{- Restitution/co-interprétation}

L'équipe des chercheurs, s'appuyant sur le travail d'écriture et sur les échanges de l'espace collaboratif, soumettra aux co-chercheurs, à la fin de chaque phase, ses interprétations provisoires et s'en suivra un échange visant à prendre en compte les réactions des co-chercheurs.

\section{Rythme du travail}

Pour chaque phase d'écriture, l'élaboration des récits se déroulera sur 3 semaines environ, dans des échanges à l'intérieur de chaque binôme chercheur/co-chercheur. Chaque binôme déterminera ses modalités de travail et la fréquence de ses échanges.

S'en suivront la mise en ligne sur l'espace collectif de la plate-forme et les échanges sur l'espace forum pendant une quinzaine de jours. 
Une semaine sera ensuite consacrée à un travail commun de co-interprétation des données réunies pendant la phase d'écriture des récits.

\section{NOTES}

1. Cette expression correspond à la prise en charge de l'individu, par lui-même, de sa destinée médicale, familiale et sociale, professionnelle et économique. (Bassleer, Boulanger, Menozzi, 2016)

2. Jacques Rhéaume (2019, p.127) considère l'empowerment comme une « réappropriation de son pouvoir », favorisé par « un travail de conscientisation, de réflexivité critique des groupes et des collectifs sur leur place actuelle et possible dans la société, de débusquer les sources des rapports d'inégalités sociales et de domination [...] ». Pour lui, « Dans le domaine des histoires de vie et de la recherche biographique en éducation, les récits de vie collectifs s'inscrivent dans cette perspective plus générale ».

3. Ardoino propose de distinguer l'implication, qui exclut une volonté consciente de l'engagement, qui nous semble plus favorable à une position d'auteur (du récit et de leur parcours).

4. Cela fut le cas aux Huitièmes rencontres Réh@b' "Réhabilitation : l'expérience » qui s'est tenu à l'UCO d'Angers du 5-6 juin 2014 et donnant la parole à des malades, ou encore le 15 juin 2016, lors d'une journée d'étude organisée des Chantiers du CIRBE, à l'occasion de la table ronde «Épreuves de la maladie » qui s'est déroulée à l'UPEC.

5. Cette proposition de Rochex réfère au travail du chercheur-clinicien, mais semble transposable au chercheur inscrit dans le champ de la recherche biographique en éducation. Celui-ci s'inscrit en effet dans une démarche similaire à celle de la clinique dans la mesure où elle produit des connaissances se présentant comme un savoir du singulier ou du particulier et se centre sur la dynamique des processus à l'œuvre, en recherchant des effets plutôt que des causes, dans une présence à soi constituée par l'expérience intersubjective et qui implique de poser le sujet en tant que sujet de son énonciation, l'invitant à la production d'un récit qui s'élabore en recréant la situation et en l'actualisant dans une relation où se déploient un soin et une écoute de l'autre attentifs à ce qui se joue pour la personne d'une quête des formes et du sens de ses expériences.

\section{RÉSUMÉS}

Les problématiques liées à la maladie chronique impliquent la mise en œuvre de recherches compréhensives innovantes pour mettre au jour et rendre compte de l'expérience sensible des malades. La recherche concernée par cette contribution, inscrite dans la perspective de la recherche biographique en éducation, visait notamment, en s'appuyant sur leur subjectivité (Thievenaz, \& Tourette-Turgis, 2015), à identifier et caractériser les handicaps et les ressources de malades atteints de maladies systémiques auto-immunes rares dans le but d'améliorer leur prise en charge et d'élargir les propositions en termes d'éducation thérapeutique. Elle a pris appui sur la mise en récit des expériences de malades, par le biais d'une e-plate-forme collaborative envisagée comme favorable au partage et à la co-construction de savoirs. Il sera rendu compte des choix méthodologiques mis en œuvre et de leurs évolutions au fil de la 
recherche pour tenter d'aller vers une compréhension et une restitution sensibles et respectueuses de l'expérience des participants.

The issues related to chronic disease involve the implementation of comprehensive innovative research to update and report on the sensitive experience of patients. The research concerned by this contribution, included in the perspective of biographical research in education, aimed in particular, by relying on their subjectivity (Thievenaz J., \& Tourette-Turgis, C., 2015), to identify and characterize the handicaps and the resources of patients with rare autoimmune systemic diseases. The aim was to improve their care and broaden the offerings in terms of therapeutic education. It was based on the narration of the experiences of patients, through a collaborative eplatform considered as favorable to the sharing and co-construction of knowledge. The methodological choices and their evolutions will be reported over the course of the research in an attempt to move towards a sensitive and respectful understanding and restitution of the participants' experience.

INDEX

Mots-clés : maladie chronique, méthodologie, pouvoir d'agir, co-construction des savoirs

Keywords : chronic disease, methodology, power to act, co-construction of knowledge

\section{AUTEUR}

\section{ANNE DIZERBO}

Docteure en Sciences de l'éducation, chercheure associée au CREAD de l'université de Rennes et à EXPERICE de l'université Sorbonne Paris Nord. Responsable du Pôle Initiatives en recherche biographique du Collège International de Recherche Biographique en Éducation 